\title{
El impacto de la economía 4.0 sobre las condiciones de trabajo y empleo. Estudio de caso en dos empresas de base tecnológica ${ }^{1}$
}

\author{
María Luz Rodríguez Fernández²; Daniel Pérez del Prado ${ }^{3}$
}

Recibido: 13 de junio de 2017 / Aceptado: 13 de diciembre de 2017

Resumen. Este artículo analiza, desde una perspectiva práctica, los cambios en las condiciones de trabajo que están teniendo lugar en las empresas que más de cerca están viviendo la era de la digitalización. Para alcanzar este objetivo, se empleará un enfoque metodológico que integra el análisis interdisciplinar, tanto jurídico como sociológico, con el estudio de caso. Los principales resultados destacan el éxito del sistema educativo para proporcionar la formación básica que este sector necesita y el correlativo fracaso del sistema de formación para el empleo; el uso extremadamente flexible del tiempo de trabajo, lo que tiene claras repercusiones en materia de conciliación; la altísima rotación laboral; y la ausencia de eficacia práctica de ciertas instituciones clásicas, como el contrato indefinido o la representación legal de los trabajadores.

Palabras clave: Digitalización; Formación; Condiciones de trabajo; TIC; Tiempo de trabajo; Conciliación vida laboral y familiar; Salarios

\section{[en] Impact of the economy 4.0 on working and employment conditions. A case study in two technological based companies}

\begin{abstract}
This paper aims to analyse, from a practical perspective, the changes on working conditions which are now taking place in the companies that are living closely the era of digitalization. In order to achieve this objective, it uses a methodological approach which integrates interdisciplinary analysis, both legal and sociological, and the so-called "case study". The main results highlight the success of educational system in providing basics skills, whereas public vocational training programs seems to be not so useful; the extremely flexible use of working time, which has clear negative effects on reconciliation; the extraordinarily high job rotation; and the lack of real effectiveness of some classical institutions, such as the indefinite contract or employees' representation.

Keywords. Digitalization; Vocational training; Working conditions; ICT; Working time; Reconciliation of work and family life; Wages.
\end{abstract}

Sumario. 1. Razones para conocer qué está sucediendo en las empresas de base tecnológica. 2. Condiciones de trabajo y empleo afectadas por la digitalización. 3. Metodología de la investigación. 4. Principales hallazgos en las entrevistas en las empresas M y B. 5. Conclusiones. 6. Bibliografía

\footnotetext{
Este artículo recoge las conclusiones preliminares del estudio "El impacto de la economía 4.0 sobre las instituciones del Derecho del Trabajo", financiado por la Fundación para el Diálogo Social.

2 Profesora Titular de Derecho del Trabajo y de la Seguridad Social en la Universidad de Castilla-La Mancha, MariaLuz.Rodriguez@uclm.es

3 Profesor Acreditado Titular de Derecho del Trabajo y de la Seguridad Social en la Universidad Carlos III de Madrid. daniel.perez.delprado@uc3m.es
} 
Cómo citar: Rodríguez Fernández, M.L.; Pérez del Prado, D. (2018) "El impacto de la economía 4.0 sobre las condiciones de trabajo y empleo. Estudio de caso en dos empresas de base tecnológica". Cuadernos de Relaciones Laborales, 36(2), 355-372.

\section{Razones para conocer qué está sucediendo en las empresas de base tecnológica}

El Foro Económico Mundial advertía en un informe reciente que, entre los años 2015 y 2020, la digitalización de la industria puede conllevar la desaparición de 7,1 millones de puestos de trabajo y la creación de 2,1 millones de nuevos empleos. Este mismo informe calcula que el $65 \%$ de los actuales estudiantes de primaria tendrán una ocupación que hoy no existe (World Economic Forum, 2016: 3, 13). La digitalización de la economía supone, así, un reto para las sociedades actuales, no solamente por la profunda transformación que implica en numerosos ámbitos y, en particular, en el de las relaciones laborales, sino por el potencial impacto que puede tener sobre el empleo.

Es verdad que la literatura sobre cómo serán (presumiblemente) el trabajo y el empleo en la era digital empieza a ser copiosa. También lo es que contamos ya con análisis de notable calidad que intentan idear los cambios que se habrán de producir en el Derecho del Trabajo a raíz de la creciente digitalización de los procesos productivos. Sin embargo, nosotros creemos que, antes de lanzarse a la creación de nuevas instituciones o la remodelación de las existentes, conviene conocer en profundidad y de primera mano qué está sucediendo en la realidad productiva de empresas y respecto de trabajadores que ya están viviendo en la era de la digitalización.

Es indudable que la cuarta revolución industrial o, según otras versiones, la segunda machine age (Brynjolfsson y McAfee, 2014), ha comenzado hace tiempo y que hay ya un número importante y creciente de empresas y de trabajadores que no tienen que esperar al futuro, sino que producen, trabajan y viven día a día bajo las condiciones que supone la digitalización. De ahí que el propósito del presente artículo sea mostrar, desde una perspectiva eminentemente práctica, las transformaciones que están experimentando las condiciones de empleo y de trabajo en esas empresas y respecto de esos trabajadores. Para ello se partirá de una base metodológica que integra el análisis interdisciplinar, jurídico y económico, y el de estudio de caso, a través de los datos extraídos de experiencias reales de dos empresas del sector tecnológico de nuestro país. Todo ello con el propósito de poder contribuir, con datos objetivos, a delimitar las tendencias de futuro de las instituciones laborales en el marco de este profundo proceso de transformación económica.

\section{Condiciones de trabajo y empleo afectadas por la digitalización}

Con la finalidad de delimitar nuestro análisis, comenzamos por identificar las áreas o instituciones laborales que con mayor seguridad pueden verse afectadas por el avance de la digitalización. Informes pioneros como el Green Paper Work 4.0 (Federal Ministry of Labour and Social Affairs, 2015) avanzaban ya alguna idea al respecto de cuáles habrían de ser esas áreas ${ }^{4}$. De ellas, nosotros nos hemos centrado en las que

Ya antes se había publicado en Reino Unido UK Commission for Emploment and Skills (2014), sobre las relaciones entre los avances de la digitalización y las exigencias en materia de formación de los trabajadores. 
guardan relación con las condiciones de trabajo y empleo, dejando al margen aquellas otras relativas a instituciones como la figura del trabajador o del empleador, las medidas de acción colectiva o la protección social, dada su falta de idoneidad para testarlas en entrevistas a trabajadores y empresarios.

1. Empleabilidad y formación: aunque existe literatura que afirma que más del $55 \%$ de los actuales puestos de trabajo de nuestro país son susceptibles de automatizarse (Berger y Frey, 2016: 17), no creemos posible que nadie pueda acertar por completo a la hora de evaluar cuántos y cuáles serán los puestos de trabajo que habrán de desaparecer a consecuencia de la digitalización; no obstante, sí puede darse por cierto que habrá destrucción de empleo y una reconfiguración de los empleos que se mantengan o puedan crearse. Ambos fenómenos pueden ser especialmente problemáticos para España. Primero porque somos ya de por sí un país con un alto volumen de desempleo. Según los últimos datos de la Encuesta de Población Activa (Instituto Nacional de Estadística, 2017), el número de personas desempleadas en nuestro país supera los 3,7 millones, con una tasa de desempleo del $16,38 \%$, la segunda más alta de toda la Unión Europea. De esta forma, sin haber recuperado todavía el empleo destruido durante la crisis económica que dio comienzo en 2008, las pérdidas de empleo que hayan de sumarse por causa de la digitalización pueden suponer alcanzar cifras de desempleo claramente intolerables.

En segundo lugar, y especialmente, porque las características de nuestra fuerza de trabajo van a hacer que sea ingente el número de trabajadores que van a necesitar formación para no perder la "carrera" frente a la tecnología. En la actualidad, la OCDE cifra en 10 millones de adultos en edad de trabajar el número de personas que en España necesitan cualificación para ocupar los empleos que puedan crearse en el futuro (Organización para la Cooperación y el Desarrollo Económico, 2015: 19). Somos, además, uno de los países que forman parte de esta Organización con una fuerza de trabajo con menor nivel de estudios. Casi el 43\% de nuestra población adulta no posee más que estudios de educación primaria, cuando la media con respecto a este nivel de los países de la OCDE es del 23\% y de la Unión Europea es del 21\% (Ministerio de Educación, Cultura y Deporte, 2016: 7).

En materia de formación digital las cifras no son mucho mejores. De acuerdo con el Informe sobre el Progreso Digital en Europa (Comisión Europea, 2017), únicamente el $53 \%$ de la población de nuestro país posee competencias digitales básicas. La media de la Unión Europea es del 56\%, pero hay que destacar dos aspectos: el primero es que, en lugar de mejorar, el último año ha descendido el porcentaje de personas con estas competencias; el segundo es que hay países que alcanzan un $68 \%$ (Alemania), 69\% (Reino Unido) o incluso 73\% (Finlandia) de sus respectivas poblaciones con competencias digitales básicas, de hecho España ocupa el puesto número 15 del total de países de la UE. Algo similar sucede en relación con las personas especialistas en tecnologías. En España son un 2,4\%, mientras que hay países donde los especialistas en TIC suponen el 5\% (Reino Unido) o incluso el 6,5\% (Finlandia) de la población en edad de trabajar. Por cierto, en esta variable también hemos empeorado el último año (del 3,1\% hemos pasado al $2,4 \%$ ) y hemos descendido al puesto número 21 de los países de la UE.

Ello pone sobre la mesa la idoneidad de la política educativa de nuestro país y la pregunta de si estará preparada para afrontar el reto de la creciente digitalización de nuestra economía. Pero también interpela directamente al sistema de formación 
para el empleo, dado que las políticas de mejora de la empleabilidad y de formación a lo largo de la vida van a adquirir una importancia central. En este sentido, se hace precisa una reflexión sobre la conformación de las políticas activas de empleo, especialmente las destinadas a la cualificación y reciclaje de la fuerza de trabajo, hoy -al menos esa es nuestra opinión- ampliamente ineficaces, y la creación de un marco que permita hacer realidad la formación a lo largo de la vida laboral, hoy prácticamente inexistente (Rodríguez, 2017).

2. Tiempo de trabajo: la digitalización hace que se pueda trabajar en cualquier tiempo y lugar y, por ello, que puedan romperse las fronteras clásicas del tiempo y el lugar de trabajo. En teoría, ello puede facilitar la combinación de los momentos laborales y de vida privada, una de las necesidades más sentidas por las nuevas generaciones de trabajadores (mujeres y hombres), lo que podríamos denominar el deseo de "soberanía" sobre el tiempo de trabajo. Pero también posibilita el trabajo en todo momento y en todo lugar, sin apenas límites y, así, sin tiempo para la vida privada y/o el descanso.

En efecto, todos los indicios apuntan a que, en lugar de gobernar con autonomía el tiempo de trabajo y el tiempo dedicado al descanso y/o cuidados, los trabajadores digitales prolongan su jornada de trabajo más allá de los estándares de los trabajadores convencionales. De hecho, y en relación con nuestro propio país, un $64 \%$ de los españoles afirman que desarrollan trabajo en las horas libres y un $68 \%$ confirman que reciben mensajes de correo electrónico o llamadas de trabajo fuera del horario laboral (Eurofound y ILO, 2017: 21-25).

De la misma forma, hay un efecto de "borrado" o dilución de las fronteras entre los tiempos dedicados al trabajo, al ocio y/o al cuidado (Webster y Randler, 2016: 1314). Muchos trabajadores atienden necesidades personales mientras trabajan; otros tantos trabajan durante sus tiempos de ocio; tiempo de trabajo pagado y tiempo de trabajo de "consumo" no pagado se confunden (Huws, 2014: 169-172); y todo ello difumina los márgenes del concepto y la medición de la jornada de trabajo. De ahí la necesaria reflexión sobre las bondades de la digitalización en relación con la libertad del trabajador respecto de su tiempo de trabajo, pero también sobre los límites que deban ponerse (legal o convencionalmente) para evitar las situaciones de sobrecarga o de falta de desconexión de las obligaciones laborales durante los tiempos de vida privada (Eurofound y ILO, 2017: 50-51).

3. Conciliación de la vida profesional y personal: la mayor flexibilidad que la digitalización podría propiciar en la combinación de la vida profesional y privada necesita ejercitarse en condiciones que la hagan posible, teniendo en cuenta, además, las necesidades organizativas de las empresas. En este sentido, debe considerarse que muchas de las actividades o tareas del trabajo digital no están sometidas a los parámetros de tiempo y lugar de una determinada localización geográfica, sino que el horario del mundo es a su vez el horario al que necesitan adaptarse muchos de estos trabajadores. La facilidad con que se diluyen las fronteras de los tiempos de vida y trabajo en esta clase de trabajos impiden no pocas veces que la conciliación sea una opción sin tensiones, dado que el sentimiento de falta de atención a las necesidades familiares en momentos pensados para ellas que son "inundados" por el trabajo digital producen sentimientos de culpa y frustración (Webster, 2016: 60). 
Por otra parte, y aunque en España apenas se haya manejado, quizá fuera de utilidad atender al concepto o perspectiva de "hora punta de la vida" (rush hour of life) como factor de distribución vital del tiempo de trabajo (Federal Ministry of Labour and Social Affairs, 2015: 20). Este concepto trata de adaptar el tiempo de trabajo a los avatares de la vida privada, en el entendimiento de que hay momentos en el discurrir de la vida en que, por edad o falta de responsabilidades familiares, el trabajador puede estar disponible para realizar un mayor tiempo de trabajo y otros en los que, también por edad y por querer asumir responsabilidades familiares, el trabajador puede estar menos disponible para la prolongación de su jornada laboral. Una distribución del tiempo de trabajo más acorde con las necesidades vitales podría ayudar a una mejor planificación de la maternidad/paternidad y, por esa razón, a un aumento de la natalidad. Una exigencia esta cada vez más imperiosa en nuestro país, con una de las tasas de natalidad más bajas del mundo, que pone en riesgo la reproducción de la fuerza de trabajo o la propia sostenibilidad de nuestro sistema de pensiones.

El teletrabajo es, para terminar con este punto, una opción que consentiría un mejor acomodo de tiempos de trabajo y tiempos privados de ocio y/o cuidado. Es claro que el uso de la tecnología como herramienta de trabajo permite localizar la actividad laboral en cualquier espacio o lugar. Ello potencia, al menos en teoría, la posibilidad de trabajar en o desde el domicilio del propio trabajador, ahorrando tiempos de desplazamiento entre el domicilio y el lugar de trabajo al mismo y el coste de los espacios físicos de trabajo a las propias empresas. De hecho, los lugares de trabajo de las empresas digitales han cambiado profundamente, dado que ya apenas existen puestos o lugares destinados a un trabajador en particular, sino espacios de uso común e indistinto por diferentes trabajadores. Sin embargo, la utilización del teletrabajo no deja de tener contraindicaciones. La primera es la que procede de los propios trabajadores, que pueden entender el trabajo en casa como una forma de aislamiento, soledad o falta de socialización (Parlamento Europeo, 2015: 27-28). La segunda guarda relación con la cultura empresarial. España es un país donde, según los informes más recientes, apenas crece el teletrabajo, dado que los empresarios cultivan el "presentismo" de la fuerza laboral y sienten la necesidad de verificar de primera mano el cumplimiento por parte del trabajador de su prestación de trabajo (Eurofound y ILO, 2017: 10).

4. Aspectos de seguridad y salud en el trabajo, especialmente riesgos psicosociales: la posible ruptura de las fronteras del tiempo y el lugar de trabajo que se anotó anteriormente puede dar lugar a una sobrecarga de trabajo y de responsabilidad que afecte a la salud de los trabajadores. El trabajo con tecnologías puede suponer problemas para la vista y/o ergonómicos (European Agency for Safety and Health at Work, 2015). Se trata de riesgos laborales clásicos, de más difícil prevención y control si el trabajo digital se realiza fuera de los centros de trabajo. Al lado de ellos se encuentran los riesgos psicosociales. La mezcla entre tiempos de trabajo y privados, la prolongación de las jornadas de trabajo, la necesidad de estar conectado en todo momento y lugar para atender las obligaciones laborales, etc., como rasgos propios de muchos de estos trabajado producen no pocas veces cansancio, estrés y el síndrome del burnout o "quemado" (Huws et al., 2016: 16-20). Todos estos aspectos deben ser convenientemente analizados y conjurados para preservar el derecho a la salud y la seguridad de los trabajadores digitales. 


\section{Metodología de la investigación}

Una vez perfiladas las áreas donde íbamos a medir el impacto en las condiciones de trabajo y empleo de la digitalización, iniciamos un doble camino.

De una parte, elegimos dos empresas de base tecnológica radicadas una en Madrid (M) y otra en Barcelona (B). Dos empresas de tamaño medio, ambas dedicadas a la consultoría tecnológica, con proyectos nacionales e internacionales y una fuerza de trabajo compuesta en su mayor parte por jóvenes ingenieros. La idea era realizar dos cuestionarios en cada una de las empresas: uno dirigido a los responsables de recursos humanos y el otro dirigido a los trabajadores representativos (nivel de educación y edad) de ambas plantillas. Se trataba de comparar las respuestas obtenidas en ambos contextos empresariales, de manera que nos permitiera contar con una primera aproximación de sus similitudes y/o divergencias.

De otra parte, confeccionamos los dos cuestionarios con preguntas que podían darnos idea de cómo estaban siendo los principales aspectos de las relaciones laborales en ambas empresas. Son dos cuestionarios en paralelo, pero con enfoques diferentes. El dirigido a los responsables de recursos humanos tiene por finalidad detectar hasta qué punto las normas y las instituciones laborales en vigor se adaptan a las necesidades que impone la dirección de una empresa prototipo del trabajo en la era digital. En cambio, el cuestionario dirigido a los trabajadores está enfocado de manera que nos permita conocer cuáles son las condiciones de trabajo de esta clase de trabajadores, las reivindicaciones que tienen en relación con ellas y la forma de gestionar (individual o colectivamente) esas mismas reivindicaciones.

No obstante, a pesar de este distinto enfoque, las áreas temáticas tratadas fueron idénticas en cuanto a su contenido para trabajadores y empresarios, de tal forma que nos permitiera realizar la correspondiente comparación no solo entre las dos empresas, sino también entre la respuestas de trabajadores y empresarios frente a un mismo tema. En concreto, se realizaron preguntas en torno a la i) la empleabilidad y la formación, (ii) tiempo y lugar de trabajo, (iii) conciliación de la vida personal y familiar, (iv) representación de los trabajadores, (v) adaptabilidad del marco normativo y condiciones salariales.

\section{Principales hallazgos en las entrevistas en las empresas M y B}

1. Por lo que hace a la empleabilidad y la formación, aunque los estudios que se han realizado hasta la fecha suelen abordar el análisis desde una perspectiva general o, si se nos permite, macroeconómica, lo cierto es que buena parte las líneas de tendencia apuntadas en ellos pueden corroborarse a la luz de los casos estudiados en el presente artículo. En concreto, las entrevistas muestran muy a las claras los avances continuos y a un ritmo acelerado que experimenta el sector, lo que exige dotar a sus trabajadores de una cualificación permanente, especializada y particularizada.

Así lo pone de manifiesto el profundo cambio en materia formativa detectado por los representantes de la empresa $\mathrm{M}$, que subrayaron que se ha pasado de una estrategia en la que se ofrecía formación amplia y de contenido genérico a otra mucho más circunscrita a determinados empleados, de alta calidad y muy focalizada en determinadas aspectos o proyectos. En este sentido, tanto la empresa M como la empresa B 
valoran la formación del sistema educativo y, en particular, del de formación profesional como adecuada en lo que hace a sus necesidades básicas. No obstante, junto a esto, también ponen de manifiesto que resulta imprescindible que ellos mismos ofrezcan la formación especializada indispensable para adquirir las certificaciones necesarias para un trabajo de alto rendimiento en el sector.

Al margen quedaría la formación ofrecida por la Fundación Estatal para la Formación en el Empleo. Tanto la empresa M como la B manifiestan su irrelevancia: únicamente les es útil la financiación (formación bonificada), pero no las actividades formativas porque el nivel que ellas requieren es muy superior al ofrecido por esta Fundación. Además, la propia financiación es insuficiente para el volumen de cursos especializados que necesitan.

Dentro de la formación ofrecida por las propias empresas suelen distinguirse dos tipos. De una parte, la formación externa cualificada. Se trata de formación técnica para trabajadores altamente cualificados y capaces de superar con éxito un curso que dará pie a una certificación. La formación suele ofrecerse por empresas privadas externas y es financiada al $100 \%$ por la empresa. No obstante, también es posible que el propio cliente ofrezca dicha formación. Supone un esfuerzo para ambas partes. Para la empresa, por el desembolso económico que implica (lo cual fue puesto de manifiesto por ambas empresas); para el trabajador porque, aunque el curso se desarrolle durante el tiempo de trabajo, debe estudiar fuera de él para obtener la certificación, lo cual implica lógicamente el empleo su tiempo personal.

De otra parte, la formación interna, que tiene, a juicio de la empresa $\mathrm{M}$, un valor equiparable a la externa, ya que ofrece el mismo nivel de cualificación y particularización. Consiste en que es la propia empresa la que ofrece la formación a determinados trabajadores y es impartida por otros de mayor experiencia y va siempre vinculada a proyectos concretos. También aquí se incluye la que deriva de los propios equipos de trabajo. Esta ha sido destacada también por los trabajadores de la empresa B, que desarrollan técnicas de enseñanza grupal o mutua.

En este mismo ámbito, un problema central del sector, puesto de manifiesto por ambas empresas, es el de la pérdida de la inversión en formación como consecuencia de la alta rotación. Este problema no parece estar siendo, sin embargo, un impedimento para que las empresas inviertan en formación, pero sí es un elemento que les preocupa. No obstante, la empresa B parece asumirlo como un coste irrecuperable; mientras que la empresa $\mathrm{M}$ está buscando soluciones legales a través de la firma de cláusulas de permanencia durante un tiempo determinado (un año) después de la formación, con la penalización del pago de determinadas cantidades en caso de incumpliendo. En el lado contrario, ni los trabajadores de la empresa M, ni de la empresa $\mathrm{B}$, perciben su marcha como algo inusual o que ello produzca un perjuicio a la empresa. Más bien al contrario, en este último caso se subraya que la empresa siempre se beneficia de la formación que reciben, con independencia del momento en que se marchen. Sea como fuere, la causa de la marcha suele encontrarse más en las condiciones de trabajo y la proyección de futuro que en la formación.

Desde un plano más competitivo, la formación de los empleados se utiliza como señalización de la empresa de cara al cliente. Contar con personal técnico en determinadas materias o proyectos es un elemento distintivo respecto de otros competidores. La dificultad estriba, según la empresa $\mathrm{B}$, en las dificultades que existen para encontrar perfiles lo suficientemente formados en alguna de las especialidades que necesitan. De acuerdo con sus datos, el $20 \%$ de los puestos no pueden cubrirse por- 
que no hay candidatos que posean las cualificaciones exigidas. Desde la perspectiva del trabajador, la formación técnica y particularizada les permite distinguirse también de otros trabajadores y emplear ese plus para empoderarse a fin de mejorar sus condiciones de trabajo.

Por último, en relación con quién determina los contenidos formativos, la empresa $\mathrm{M}$ señala que son los propios trabajadores los que sugieren su itinerario formativo. Sin embargo, sus trabajadores afirman todo lo contrario, que no tienen papel alguno en materia formativa y que los cursos que se ofrecen únicamente se basan en las necesidades de la empresa en el corto plazo. Es, a su juicio, el mercado el que demanda $\mathrm{y}$, por tanto, determina el tipo de formación que se imparte. En este sentido, no creen que la formación sea la adecuada para sus necesidades, sino que es la necesaria para la prestación de un servicio o proyecto determinado en un momento determinado. Esta diversa valoración de la formación que se ofrece, unido a las bajas condiciones de trabajo, son factores que explican la alta rotación del sector, a la que aludiremos más adelante.

Por su parte, la empresa B y sus trabajadores corroboran el carácter unilateral de la formación que se imparte en el sector, de tal forma que corresponde a la empresa, en su mayor parte, diseñar los itinerarios formativos de sus empleados. No obstante, en esta misma empresa se señala, de forma contradictoria, que corresponde a los trabajadores diseñar su itinerario profesional, que ella decide si apoyará. Además, en su opinión, cuanto más grande es la empresa, menos capacidad tiene el trabajador a este respecto.

2. Por lo que hace al tiempo de trabajo, resulta difícil hacer una caracterización general. Y ello por dos razones. De una parte, porque la jornada, el horario y los turnos varían en función de la tarea que se desarrolle y el departamento en el que se trabaje. De otra, porque incluso en los supuestos más estables, se observa una cierta flexibilidad en cuanto a los horarios y número de horas trabajadas. De hecho, quizá el denominador común del sector, cualquiera que sea la empresa, el tipo de trabajador o la tarea que se desarrolle, es precisamente el de la flexibilidad en este campo. Esto conectaría con la descripción teórica que han puesto encima de la mesa algunos estudios a los que aludimos anteriormente: la digitalización implica una tendencia hacia el trabajo en todo momento y en todo lugar, sin apenas límites o con unos límites ciertamente difusos.

Ello se manifiesta en la ausencia de cualquier tipo de norma tendente a regular jornada y horario, la necesidad de apartarse del corsé del convenio sectorial y la preferencia por su encauzamiento a través de prácticas de empresa. Así, la empresa $M$ señala que hay flexibilidad de entrada y salida, aunque no existe regulación aplicable y en última estancia reconocen que tal posibilidad depende del proyecto en que se trabaje. La jornada continua durante el verano también ha sido aplicada de una forma espontánea o informal. Los trabajadores de la empresa B practican la jornada estable salvo determinadas excepciones puntuales. Esta sería una tendencia general, la de gozar de un cierto marco mínimo estable, proporcionado por el convenio o los acuerdos individuales, que es flexibilizado o adaptado al margen de su regulación formal en función de las necesidades de la empresa.

En materia de desconexión tecnológica, ni los trabajadores de $\mathrm{M}$, ni los trabajadores de B, manifiestan tener especiales problemas a este respecto (si bien esto depende de la división, puesto que los trabajadores de "sistemas", a los que se imponen 
determinados turnos, sí que parecen sufrirlo tal y como vamos a ver a continuación), ni que hayan tenido que trabajar los fines de semana. Con carácter general, gozan de cierta autonomía para administrar su tiempo de trabajo, lo cual es corroborado tanto por los representantes de la empresa M, como de B. Preguntados por la desconexión, los dirigentes de la empresa $\mathrm{M}$ apuestan más por la autorregulación de esta cuestión que por la imposición legal, como en el caso de Francia.

Por último, es preciso señalar la importancia que en este sector tienen los turnos especiales, lo que conecta directamente con el tipo de servicios que se prestan o, si se prefiere, con las demandas del mercado. Así, en ambas empresas existe el llamado "turno 24x7", que se vincula a proyectos e implica atención continuada al servicio las veinticuatro horas del día, los siete días de la semana, compensándose al trabajador mediante un suplemento económico. La empresa B, no obstante, señala que su aplicación es minoritaria, sólo para algunos trabajadores de plantilla.

Para ambas empresas, los turnos se establecen a través de negociación individualizada con el trabajador o, en último caso, es decidida unilateralmente por la empresa. Los trabajadores de la empresa $\mathrm{M}$ muestran este tipo de turnos como la regla general en determinadas áreas de la empresa y los conectan a las propias demandas del mercado y, en última instancia, de la sociedad. Una sociedad que desea que sus servicios tecnológicos funcionen veinticuatro horas requiere en última instancia de sujetos que supervisen y controlen cómo se presta el servicio durante esas mismas 24 horas. Además, la solución de los problemas ha de hacerse en el mínimo tiempo imprescindible, lo cual está expresamente determinado en los contratos. Asimismo, advierten que este tipo de turnos serán la tónica dominante y generalizada en el futuro. De hecho, señalan como ejemplo que todos los nuevos contratos incorporan la obligación de trabajar bajo el turno $24 \times 7$ en caso de que sea necesario, frente a los contratos antiguos que preveían jornada y horarios estables.

3. Naturalmente un panorama como el descrito en materia de tiempo de trabajo va a tener su lógica traslación en el ámbito de la conciliación de la vida personal y familiar. Para empezar, ninguna de las empresas estudiadas posee ningún tipo de regulación a este respecto. La forma de compaginar la jornada y horarios flexibles con la conciliación es su reparto entre los trabajadores integrantes del equipo, negociándose en su seno y/o con el jefe de equipo, decidiendo éste en última instancia. Esta forma de actuación es común a ambas empresas. No obstante, la situación varía, como en casos anteriores, en función del servicio y de la antigüedad.

Estas mismas reglas se aplican en el caso de los turnos. La configuración actual de los turnos 24x7 lleva aparejada una respuesta inmediata por parte del trabajador. Esto -señalan los trabajadores de la empresa M- supone que la dependencia de la empresa es total una vez finalizada la jornada de trabajo. En su opinión, si bien hace un tiempo era posible compaginar las guardias con actividades personales, hoy ya no resulta posible, debido a los cortos lapsos de tiempo de que disponen para responder a las incidencias, lo que tiene un fuerte impacto en su vida personal. Por el contrario, los trabajadores de la empresa $\mathrm{B}$, que habían manifestado tener horarios más estables comparativamente, muestran también como consecuencia una mejor posición a la hora de conciliar. No obstante, parece que las situaciones no son homogéneas y que dependen mucho de la tarea y división en que se trabaje.

De su lado, las TIC no parecen estar propiciando una mejora en la conciliación, tal y como para nuestro país habían descrito los estudios teóricos. El teletrabajo 
no está generalizado y sólo se aplica para supuestos puntuales, salvo en el caso de los trabajadores que se encuentran en otras comunidades autónomas o trabajadores autónomos. Aunque ambas empresas manifiestan que sería posible emplearlo más, reconocen que lo ven con recelo por la falta de control sobre el trabajador, la posibilidad de ser un derecho adquirido como condición más beneficiosa (empresa M) y la necesidad de que haya interrelación y trabajo en equipo (empresa B). Tanto M como B asumen, no obstante, que para la empresa podría resultar rentable por el ahorro de costes en infraestructuras.

Frente a la clara postura empresarial, la de los trabajadores es más dispar. Para algunos trabajadores, especialmente en la empresa $\mathrm{M}$, la empresa no está llevando a los máximos límites el teletrabajo, lo que sería técnicamente perfectamente factible. Es más, a su juicio sería un complemento ideal para los turnos $24 \times 7$, pues permitirían compensar la mayor dependencia con el trabajo sin desplazamiento. Además, señalan que la única causa por la que no se desarrolla más intensamente en la empresa es que los mandos siguen con una postura tradicional de control mediante la presencia. Ni siquiera una opción mixta, mañana presencial y tarde teletrabajo, ha sido considerada. Por el contrario, otros trabajadores, especialmente en la empresa B, manifiestan no tener especiales problemas para trabajar en casa, si bien no está regulado o protocolizado. No lo ven como una posibilidad permanente, sino para momentos puntuales, por los riesgos de desconexión con la empresa y la falta de delimitación entre lo personal y lo laboral. Todos los trabajadores entrevistados en la empresa B prefieren el trabajo presencial en la empresa al teletrabajo.

4. Por último, como también quedó advertido, las profundas transformaciones que estamos viviendo ponen al descubierto la falta de adaptación del tradicional marco regulatorio laboral a algunos de los determinantes que se viven en estas empresas.

Así, ambas se quejan -insistentemente- de que algunas instituciones laborales no son flexibles y no se adaptan a las necesidades del sector. No obstante, a pesar de que el convenio colectivo sectorial lo consideran rígido, la empresa B no ve la necesidad de negociar un convenio de empresa. Su principal vía de adaptación es la negociación de las cuestiones problemáticas con el comité de empresa, con el que reconocen tener una relación fructífera; y a través de la negociación directa con los trabajadores, destacando especialmente el ámbito salarial. Esta última vía, la negociación directa con los trabajadores, parece ser también un mecanismo adecuado de adaptación a las necesidades del mercado para la empresa M, que no cuenta con representación de los trabajadores, a pesar de que legalmente estaría obligada a ello.

Esto, sin embargo, no es óbice para que, en materia de contratación, parezca existir una cierta predisposición hacia el contrato indefinido. Los responsables de la empresa B destacan que el recurso mayoritario a la contratación fija obedece al hecho de que, al encontrarse en un sector altamente competitivo, los trabajadores no admitirían otra fórmula contractual, si bien reconocen que a efectos prácticos las diferencias entre contratación indefinida y temporal no son muy relevantes desde el punto de vista empresarial.

En cuanto al resto de modalidades contractuales, ambas empresas reconocen utilizar con carácter puntual el contrato de obra, normalmente vinculado a algún proyecto; el contrato en prácticas en el caso de la empresa $\mathrm{M}$, pero no así en el de la empresa $\mathrm{B}$, que recurre a becarios; $\mathrm{y}$, más excepcionalmente aún, los autónomos. 
Tanto en el caso de los contratos en prácticas en la empresa $\mathrm{M}$, como las becas en la empresa B, son empleados como vías de entrada en la compañía. Sin embargo, la empresa B señala que le gustaría poder recurrir más al contrato de obra y a los trabajadores autónomos por la alta competitividad y el carácter cíclico de la actividad del sector. El argumento que subyace en el fondo es la necesidad de poder ajustar costes y producción a la demanda. De hecho, los responsables de la empresa B llegan a afirmar, en relación a lo primero, que consideran que los costes del despido son excesivos. La empresa M manifiesta que el contrato de emprendedores no lo han utilizado todo lo que quisieran por las bonificaciones y el límite de 50 trabajadores, que les implica un importante trastorno organizativo.

Los trabajadores de la empresa $\mathrm{M}$ y B corroboran que el contrato de uso ordinario es el indefinido, si bien no perciben que por ello vayan a gozar de una mayor estabilidad, puesto que, si en otro sitio les ofrecen mejores condiciones de trabajo o proyección profesional, se marcharán teniendo allí la misma modalidad contractual. La visión de los trabajadores, en particular en la empresa $\mathrm{M}$, es que los contratos temporales, en concreto los de obra y servicio y en prácticas (y las becas en el caso de la empresa B ), se utilizan a modo de prueba (sin perjuicio de incluir en ellos un determinado período de prueba que fijan en tres meses), lo que es corroborado por la dirección de la empresa $\mathrm{B}$ (la empresa $\mathrm{M}$ no se manifiesta al respecto). A partir de ahí lo habitual es concertar un contrato indefinido, entre otras cosas porque, como se ha señalado, los propios trabajadores no admitirían otra opción, aunque ello no implique una mayor estabilidad, como venimos insistiendo. Este elemento, el de la estabilidad, es más valorado por los trabajadores más mayores, que por los jóvenes.

En este ámbito, los trabajadores de la empresa $M$ ponen de manifiesto que las bajas condiciones laborales son una de las causas fundamentales de la alta rotación que vive el sector, lo que se sintetiza muy a las claras en la frase "ya nadie se casa con la empresa" (frase que, por cierto, también es utilizada por otro trabajador en la empresa B). De hecho, entre los factores que más valoran a la hora de cambiar de empresa se encuentra el salario, pero también horario y distancia con el puesto de trabajo en aras a una mejor conciliación. En un intento de evitar esta alta rotación, los trabajadores de la empresa M subrayan la práctica empresarial de intentar mejorar la oferta de la competidora cuando el trabajador anuncia su marcha. En este sentido, en este sector adquiere cierta importancia el precontrato como prueba cierta de que el trabajador tiene una propuesta en firme mejor por la cual se marcha.

De otra parte, la necesidad de una mayor flexibilidad en la regulación a que venimos aludiendo se traslada también al ámbito de la representación de los trabajadores. Para la empresa $\mathrm{M}$, este tipo de mecanismos no resultan necesarios porque hay un trato muy directo con los trabajadores. La regulación de las condiciones de trabajo es personalizada e individualizada. En algunos casos, como quedó apuntado, se buscan acuerdos entre los propios trabajadores y, en caso contrario, decide el superior.

No es ésta, sin embargo, la visión de los trabajadores, que, con carácter general, valoran positivamente la existencia de representación. Así, en la empresa M creen que sería imprescindible este tipo de mecanismos que permitiera la interlocución y canalización de sus reivindicaciones, en especial en relación con aquellos trabajadores que disfrutan de peores condiciones o que se encuentran en una situación más precaria. Para los trabajadores de la empresa B, que sí cuentan con representación, la importancia de la labor de sus representantes es relativa. Creen que hay materias que pueden canalizar a través de la representación unitaria, mientras que otras deben 
gestionarlas directamente con el empresario. De hecho, el grado de negociación individual es también comparativamente alto.

Quizá debido a este alto grado de individualización de las relaciones de trabajo los salarios no son todo lo bueno que querrían, lo que los representantes de las empresas achacan a la alta competencia internacional. De acuerdo con la postura de los trabajadores de la empresa $\mathrm{M}$, las condiciones salariales de los ingenieros han cambiado de una forma muy notable si se compara la situación hace años con los actuales, de tal forma que han empeorado considerablemente. A pesar de ello, fórmulas como el salario variable no parecen estar excesivamente extendidas La empresa B fija el porcentaje de trabajadores que reciben remuneración variable en torno al 10\%.

\section{Conclusiones}

A lo largo de estas páginas hemos intentado aportar, desde el estudio de casos reales, algunas líneas de tendencia que se observan en las empresas del sector tecnológico y que pueden ayudarnos a comprender en qué medida habrán de transformarse algunas instituciones del Derecho del Trabajo para hacer frente con éxito a los retos que supone la digitalización de la economía.

Una primera conclusión es la profunda dependencia que tienen las empresas tecnológicas de la formación de sus trabajadores. Como en cualquier otra clase de empresas, la calidad profesional de su fuerza de trabajo es condición necesaria para realizar con eficacia la actividad productiva a la que se dedican, pero a estas empresas la especialización de sus trabajadores les sirve también como elemento reputacional para posicionarse en el mercado. Dada la escasez de expertos en determinados ámbitos del trabajo tecnológico, tener en platilla a especialistas en tales campos supone situarse como empresa en la vanguardia del sector y, por ello, ser más competitiva que las demás, en un sector que es altamente competitivo (recordemos que estas empresas compiten a escala internacional porque la tecnología y el uso del inglés como lengua oficial del sector facilitan que puedan prestar servicios en cualquier lugar del planeta).

En esta materia de la formación, otra de las conclusiones es que aprueban con nota el sistema educativo y el sistema de formación profesional y suspende, sin embargo, el sistema de formación para el empleo. A pesar de que todas estas empresas utilizan prácticas, becas y contratos en prácticas (la verdad es que más prácticas y becas que contratos en prácticas) como elementos de transición entre el sistema educativo y el trabajo, tienen una valoración altamente positiva de los conocimientos de base que tienen los aspirantes a trabajadores. No sucede lo mismo con el sistema de formación para el empleo.

Es ciertamente paradójico que un sistema que está pensado para ayudar a las empresas a proporcionar a sus trabajadores formación permanente apenas sea utilizado en unas empresas que demandan genéticamente una continua actualización de la formación de sus trabajadores. El sistema de formación para el empleo se utiliza como simple mecanismo de abaratamiento de costes (a través de la formación bonificada), pero en ámbitos claramente laterales o marginales de la formación que requieren estos trabajadores. Ello genera un sentimiento que podríamos denominar de "desapego empresarial" respecto del sistema. Lo verdaderamente relevante en materia de 
formación se produce al margen del mismo: es la empresa el centro de gravedad de la formación de los trabajadores. Es ella la que decide qué formación necesitan, la que asume el coste de la formación y la que asume también el riesgo de que, una vez adquirida la misma, los trabajadores se vayan y empiecen a trabajar con una empresa competidora (la falta de una regulación que proteja los intereses empresariales frente a estas prácticas y el desapego de los trabajadores con respecto a las empresas donde trabajan son dos de las conclusiones evidentes de esta investigación).

En materia de tiempo de trabajo resultan evidentes tres conclusiones. La primera es que las normas que regulan esta institución, sean las de origen legal, sean las de origen convencional, son irrelevantes, en el sentido de que apenas se aplican; la segunda es que el tiempo de trabajo supera en muchas de las ocupaciones del sector el considerado estándar o habitual (si por tal entendemos jornadas diarias de 8/9 horas y la semanal, mensual y anual correspondiente a este multiplicador); la tercera es que el tiempo de trabajo es cuestión de "arreglo individual". Pero vayamos por partes.

Aunque algunos programadores tienen horarios estables y razonables en cuanto a su duración, lo habitual es que el horario y el tiempo dedicado a trabajar dependa de las necesidades del proyecto, teniendo en cuenta, además, que, como dijimos antes, el proyecto puede desarrollarse en cualquier zona horaria del mundo. Es claro que, ante estas necesidades productivas, que no son impostadas sino reales, las normas que en la actualidad regulan la jornada de trabajo y la determinación del horario no resultan adecuadas para esta clase de empresas. Pero de ahí a la absoluta anomia que en materia de tiempo de trabajo parece reinar en el sector hay un largo camino.

Primero porque no es infrecuente el esquema de trabajo $24 \times 7 y$, aun en supuestos donde no se sigue este esquema, es más que habitual la jornada por encima de las 10 horas de trabajo diarias y la posibilidad en algunas de las ocupaciones de tener que estar alerta o conectado fuera del horario de trabajo. Cierto es que respecto del tiempo de trabajo existe una notable ambivalencia por parte de los propios trabajadores. La autonomía en la gestión de su propio tiempo y desempeño es un valor para muchos de ellos, sobre todo cuando están ocupándose de proyectos que pueden servirles para revalorizar su propia carrera profesional sumado a su juventud (repárese en que la edad de los senior del sector está en torno a 40 años).

Pero al lado del anterior aspecto positivo hemos encontrado síntomas de un trasfondo que no lo es tanto. Confesiones de tensión o estrés por acumulación de trabajo y falta de descanso; reivindicaciones de jornada continuada durante los meses de verano; reclamaciones de un mayor tiempo de teletrabajo; reconocimiento de la necesidad de autocontrolarse los fines de semana para no responder mensajes que llegan desde la empresa. Todo ello son indicios -y esta es nuestra siguiente conclusión-, si no de malestar, sí de que la autonomía sobre la gestión del tiempo de trabajo es más pretendida que real y de que, ante la falta de aplicación de los referentes normativos en materia de jornada de trabajo, la consecuencia es que la excesiva prolongación del tiempo de trabajo se convierte en una regla del trabajo en el sector.

Es verdad que todavía no parecen estar maduras alternativas como el derecho a la desconexión de la experiencia francesa $\mathrm{a}^{5} \mathrm{o}$ el apagado de servidores de algunas

Teniendo como base el denominado Informe Mettling (2015), el legislador francés ha reconocido el derecho de los trabajadores a la desconexión digital en la Ley n ${ }^{\circ} 2016-1088$, de 8 de agosto de 2016, por medio de la inclusión de un nuevo apartado $7^{\circ}$ en el artículo 2242-8 del Código de Trabajo (https://www.legifrance.gouv.fr/ affichTexte.do?cidTexte=JORFTEXT000032983213\&categorieLien=id). 
empresas americanas. Empresas y trabajadores parecen preferir la autorregulación, sea entre los miembros del equipo que trabaja en el mismo proyecto, sea la propia autocontención en el uso del tiempo de trabajo. Pero dadas las consecuencias que produce la anomia en la gestión del tiempo en estas empresas, una reflexión sobre la conveniencia de encontrar algún mecanismo regulatorio capaz de aunar flexibilidad en relación con el quantum y la distribución del tiempo de trabajo y límites a la excesiva prolongación de las jornadas laborales parece de todo punto conveniente.

Entre dichas consecuencias se halla la dificultad para compatibilizar la vida profesional con la privada. Toda la literatura que existe al respecto apunta a esta dificultad, que nosotros hemos corroborado en la práctica. Pero antes de profundizar en este ámbito conviene hacer alguna precisión.

La fuerza de trabajo del sector es mayoritariamente masculina; hay mujeres, pero pocas. La edad media de los trabajadores del sector es baja; hay seniors, pero no son la mayoría. Esta doble característica de las plantillas en las empresas de base tecnológica hace que el tema de la conciliación entre vida profesional y privada sea visto como algo ajeno a las preocupaciones inmediatas, tanto por las empresas como por los propios trabajadores. Sin embargo, hay huellas de que la conciliación no es tarea fácil. Así, cuando preguntamos a una joven trabajadora, encantada de trabajar más de 11 horas diarias para proyectarse profesionalmente, ¿podrías tener hijos con este ritmo de trabajo? La respuesta fue no. Semejante fue la contestación de otra de las jóvenes programadoras, esta con horario más estable, sobre qué hará cuando quiera tener hijos. La respuesta: buscarme otra empresa. Igualmente asistimos a la reclamación del teletrabajo por trabajadores varones más mayores que tienen hijos a su cargo y están divorciados. Todos estos detalles indican que, llegado el deseo y/o momento de cuidar de los hijos, el trabajo en el sector de las tecnologías no lo pone fácil.

Y esto es bien paradójico porque, al menos en teoría, el uso de las nuevas tecnologías y la posibilidad de realizar la prestación de trabajo en cualquier tiempo y lugar podría consentir una mejor combinación de la vida profesional y la privada. No parece ser así. Ni siquiera lo de realizar el trabajo en cualquier lugar. Pero antes de ir con este tema, y para culminar el relativo a la combinación de tiempos de vida y de trabajo, parece que entre los límites que deberían integrarse en ese mecanismo regulatorio del tiempo de trabajo a que hemos hecho referencia habría de haber alguno que permitiera un mejor acomodo entre la vida profesional y el deseo de cuidar de los hijos alcanzada una determinada edad. Por otro lado, no estaría demás que hubiera un replanteamiento sobre el trabajo de las mujeres en este sector.

Es un hecho cierto que estudian ingeniería muchas menos mujeres que hombres. Según datos del Instituto de la Mujer (2017a), únicamente un 25,85\% del alumnado universitario que estudia ingeniería y arquitectura son mujeres. Ello explica que las plantillas de las empresas de base tecnológica sean mayoritariamente masculinas y que haya una abultada desigualdad de género en las ocupaciones de los sectores de alta y alta-media tecnología, donde las mujeres representan menos del 30\% del conjunto de la fuerza de trabajo (Instituto de la Mujer, 2017b).

No se conocen en plenitud las razones que provocan estas diferencias entre mujeres y hombres en cuanto a sus preferencias de estudios/trabajos, aunque sí se sabe que los estereotipos juegan algún rol al respecto. En este sentido, el estereotipo del trabajo en empresas de base tecnológica - masculino, con jornadas prolongadas, sin apenas tiempo de desconexión del trabajo, con dificultades para la maternidad- puede guardar alguna relación con el menor interés de las mujeres por esta clase de estu- 
dios y de trabajos. Idear mecanismos que hicieran más female-friendly los ambientes de trabajo en la empresas tecnológicas podría contribuir a que a medio plazo hubiera más mujeres estudiando ingeniería y, así, más candidatas a trabajar en el sector. Dada la escasez de fuerza de trabajo existente en el mismo, todo lo que ayudara a una mayor incorporación de mujeres a los estudios/trabajos relacionados con la tecnología sería conveniente para las propias empresas.

Uno de esos mecanismos podría ser la consideración del tiempo de trabajo desde el enfoque rush hour of life, del que no hemos encontrado rastro alguno en la realidad española, ni siquiera a nivel de simple conocimiento del fenómeno; otro el teletrabajo. En relación con esta última modalidad, nuestra investigación ha venido a confirmar las apreciaciones de la literatura especializada, esto es, que la cultura empresarial de nuestro país es eminentemente presentista y que ello es un obstáculo para la implantación del teletrabajo.

Aun reconociendo un margen de libertad para poder trabajar desde casa en algunos momentos esporádicos, la preferencia empresarial es que los trabajadores desarrollen su actividad productiva en los locales de la empresa a fin de poder controlar qué, cómo y cuándo se realiza la prestación de trabajo (el propio lugar de trabajo favorece esta supervisión, porque suele tratarse de espacios diáfanos). La afirmación de uno de los responsables de recursos humanos es bien elocuente al respecto: tienen tal conocimiento de la tecnología, que siempre podrían engañarnos sobre si están o no trabajando.

Junto a ello, hay que reconocer que dentro de los trabajadores tampoco hay una posición unitaria. Algunos, más mayores y con hijos, abogan por una mayor capacidad de trabajar desde casa, dado que gracias a la tecnología que utilizan pueden perfectamente hacerlo; pero otros, sobre todo los más jóvenes, prefieren acudir a los locales de la empresa y dejar el trabajo en el domicilio para momentos puntuales en que necesitan mayor concentración. La razón de esta preferencia es la socialización y también la separación de espacios públicos y privados. Prefieren compartir tiempo y lugar de trabajo con los compañeros que trabajar en soledad, además de que ir a la empresa ayuda a preservar como espacio para la vida privada su domicilio particular, lo que, al menos en algunos casos, resulta deseable para los trabajadores.

De lo anterior se derivan dos conclusiones. Primero: el empresariado español, aun el de empresas en la vanguardia tecnológica, sigue decantándose por la supervisión directa del desarrollo del trabajo por parte de los trabajadores. De partida, ello no es ni mejor ni peor (aunque denota desconfianza en relación con la fuerza de trabajo), pero produce consecuencias que deben ponderarse. La primera es que el tiempo que se trabaja y no el resultado del trabajo siguen siendo determinantes en la composición salarial del sector, de modo que, en lógica correspondencia, en él predominan los salarios fijos frente a los salarios variables. Lo que significa un predominio de los costes fijos para las empresas. La segunda es que con este modelo presentista estas asumen los costes de la infraestructura laboral, cuando las posibilidades de teletrabajo permitirían un importante ahorro económico por este concepto. A cambio, la presencia de los trabajadores en los locales de la empresa permite un mayor control sobre el desempeño laboral y también que estos no se desconecten del ecosistema empresarial, lo que es positivo en términos de identificación con el proyecto de la empresa.

La segunda conclusión tiene que ver con los trabajadores. Como acabamos de ver, el interés en relación con el teletrabajo depende de la edad. Los trabajadores más 
jóvenes (hombres y mujeres) prefieren el trabajo en la empresa; los más veteranos (hombres y mujeres) prefieren incrementar las posibilidades de trabajar en su domicilio. Existe, por tanto, una evidente brecha en función de la edad conectado con la necesidad de afrontar las responsabilidades familiares al llegar un determinado momento de la vida adulta, en un trabajo que se considera apasionante en términos de carrera profesional por los propios trabajadores, pero también profundamente invasivo en términos de tiempo y de falta de vida privada.

Nuestras últimas conclusiones guardan relación con una especie de ruptura generacional que hemos detectado en el sector. Para empezar, todos los trabajadores que hemos entrevistado tienen contratos indefinidos. La temporalidad existe; de hecho la forma de entrar en el sector es un mecanismo temporal: prácticas, becas, y contratos en prácticas. Por cierto, para ellos es absolutamente normal y positivo empezar de este modo a trabajar en el sector, dado que haber hecho prácticas o haber sido becario en alguna empresa puntera en tecnología es un mérito en su carrera profesional. Pero pasado este primer momento, lo habitual es que los trabajadores tengan contratos indefinidos. Sin embargo, ello no es en modo alguno símbolo de estabilidad.

Tienen contratos indefinidos porque muy probablemente no aceptarían trabajar en las empresas si se les ofreciera un contrato temporal; pero tener esa clase de contratos no les vincula a la empresa más que hasta que obtienen una mejor oferta de trabajo en términos económicos y de carrera profesional. Ambas variables tienen importancia en su toma de decisión sobre los trabajos que aceptan. Para ellos la estabilidad en el empleo no es un valor o, al menos, no tiene el mismo valor que para los trabajadores de generaciones anteriores. El salario y su carrera profesional son los vectores que realmente cuentan. Son conscientes de la escasez de mano de obra en el sector y de su propia capacidad o valía profesional y, por tanto, de que pueden encontrar un nuevo trabajo que les satisfaga más sin demasiadas dificultades. Ello hace que sea una práctica profesional habitual en el sector la alta rotación de los trabajadores y que ello suponga a veces importantes problemas para las empresas, no sólo por los costes en formación que puedan haber asumido, sino incluso porque en algunas ocasiones se preavisa la dimisión con muy poco tiempo de antelación y ello puede poner en riesgo la continuidad del proyecto comprometido con el cliente. En cambio, ello no supone ningún problema para los trabajadores, que, como ellos mismo dicen, "no se casan con nadie".

Tal vez sea por causa de los salarios. Aunque no tenemos datos concluyentes sobre la cuantía de los salarios en estas empresas de base tecnológica (algunos ingenieros hablan de 24.000 euros brutos anuales, con un salario de entrada en el sector de 15.000), sí sabemos que los pactan individualmente y que es continua la queja de que el salario ya no es el que era antes de la crisis. A lo que sumamos lo que nos han referido como práctica habitual cuando un trabajador comunica a su empresa que le han ofrecido una mejor oferta de trabajo. En estos casos, lo que hace la empresa que quiere que el trabajador se quede es mejorar económicamente la oferta de la empresa competidora. Ello significa que, aun siendo importante la proyección profesional, al final el salario es un dato fundamental para seguir en una empresa o marcharse a otra, lo que nos hace pensar que el alto nivel de rotación entre los trabajadores del sector guarda relación con los relativamente bajos salarios que se pagan en el mismo. Es sabido que en sectores con escasez de mano de obra especializada suele haber una tendencia a la subida salarial; en nuestro país, sin embargo, parece que ese poder que da la escasez de mano de obra especializada a los trabajadores que tienen esa 
especialidad, en lugar de saldarse con una subida de salarios en el sector, se salda con alta rotación laboral.

Junto con la falta de valor de la estabilidad en el empleo está la falta de interés por las instituciones colectivas de representación y defensa de los intereses de los trabajadores. Por eso hablamos de ruptura generacional. Dos elementos que son nucleares en relación con trabajadores más clásicos, resultan prácticamente irrelevantes para los trabajadores de empresas de base tecnológica. Las condiciones de trabajo (tiempo y salario) se pactan individualmente y los problemas laborales se arreglan también de forma individual. Las figuras de representación colectiva sirven a efectos de información sobre los derechos, pero poco más. Las posibilidades de negociación de un convenio colectivo apenas se contemplan (tampoco por la parte empresarial) y los conflictos más serios se dirimen con cambios de empresa por parte de los trabajadores. La devaluación de las instituciones colectivas es, por tanto, otra de las señas de identidad de estas empresas y de sus trabajadores, de modo que tenemos que preguntarnos si no caminamos hacia un modelo de relaciones laborales sin contrapesos colectivos frente al poder empresarial.

Por último, debemos hacer una advertencia. Creemos que el cuestionario que hemos utilizado nos ha permitido conocer en detalle qué sucede en la realidad de algunas empresas de base tecnológica, de manera que consideramos que es un cuestionario idóneo, en el sentido de servir para el fin que pretende. Ahora bien, somos conscientes de que la muestra de empresas y trabajadores que hemos utilizado en este estudio es muy limitada y que, en consecuencia, no podemos generalizar las conclusiones que se acaban de esbozar.

\section{Bibliografía}

Berger, T. y Carl Benedikt Frey, C. B. (2016). Digitalization, Jobs, and Convergence in Europe: Strategies for Closing the Skills Gap. http://www.oxfordmartin.ox.ac.uk/downloads/ reports/SCALE_Digitalisation_Final.pdf [consulta 13 de diciembre de 2017].

Brynjolfsson, E. y McAfee, A. (2014). The Second Machine Age, Work, Progress, and Prosperity in a Time of Brilliant Technologies. New York: Norton \& Company.

Comisión Europea (2017). Europe's Digital Progress Report 2017. https://ec.europa.eu/digital-single-market/en/scoreboard/spain [consulta 13 de diciembre de 2017].

Eurofound y ILO (2017). Working anytime, anywhere: The effects on the world of work. $\mathrm{https} / / / \mathrm{www} . e u r o f o u n d . e u r o p a . e u /$ sites/default/files/ef_publication/field_ef_document/ ef1658en.pdf [consulta 13 de diciembre de 2017].

European Agency for Safety and Health at Work (2015). A review on the future of work: online labour exchanges, or 'crowdsourcing': implications for occupational safety and health. https://osha.europa.eu/en/tools-and-publications/publications/future-work-crowdsourcing/view [consulta 13 de diciembre de 2017].

Federal Ministry of Labour and Social Affairs (2015). Re-Imagininf Work. Green Paper Work 4.0. http://www.bmas.de/SharedDocs/Downloads/DE/PDF-Publikationen/arbeiten-4-0green-paper.pdf;jsessionid=4433E1983C107164CCA2F6967AA6E758?_blob=publicationFile $\& v=2$ [consulta 13 de diciembre de 2017].

Instituto de la Mujer (2017a). Mujeres en cifras. Educación y alumnado universitario. http:// www.inmujer.gob.es/MujerCifras/Educacion/AlumnadoUniversitario.htm [consulta 13 de diciembre de 2017]. 
Instituto de la Mujer (2017b). Mujeres en cifras. Ciencia y Tecnología. http://www.inmujer. gob.es/MujerCifras/CienciaTecnologia/Empleo.htm [consulta 13 de diciembre de 2017].

Huws, U. (2014). Labor in the Global Digital Economy. The Cibertariat Comes of Age. New York: Review Press.

Huws, U., Spencer, N. H. y Joyce, S. (2016). Crord Work in Europe, Preliminary results from a survey in the UK, Sweden, Germany, Austria and the Netherlands. http://www. feps-europe.eu/assets/39aad271-85ff-457c-8b23-b30d82bb808f/crowd-work-in-europedraft-report-last-versionpdf.pdf [consulta 13 de diciembre de 2017].

Instituto Nacional de Estadística (2017). Encuesta de Población Activa, Tercer Trimestre de 2017. http://www.ine.es/daco/daco42/daco4211/epa0317.pdf [consulta 13 de diciembre de 2017].

Mettling, B. (2015). Transformation numérique et vie au travail. http://www.ladocumentationfrancaise.fr/var/storage/rapports-publics/154000646.pdf [consulta 13 de diciembre de 2017].

Ministerio de Educación, Cultura y Deporte (2016). Panorama de la educación. Indicadores de la OCDE 2016. http://www.mecd.gob.es/dctm/inee/eag/panorama2016okkk.pdf?documentId=0901e72b82236f2b [consulta 13 de diciembre de 2017].

Organización para la Cooperación y Desarrollo Económico (2015). Informe de diagnóstico de la Estrategia de Competencias de las OCDE: España. http://www.oecd.org/skills/nationalskillsstrategies/Spain_Diagnostic_Report_Espagnol.pdf [consulta 13 de diciembre de 2017].

Parlamento Europeo (2015). Employment and Skills Aspects of the Digital Single Market Strategy. http://www.europarl.europa.eu/RegData/etudes/STUD/2015/569967/IPOL_ STU(2015)569967_EN.pdf [consulta 13 de diciembre de 2017].

Rodríguez, M. L. (2017). "Plataformas, microworkers y otros desafíos del trabajo en la era digital". En L. Mora Cabello de Alba y M. L. Rodríguez Fernández (ed.), El Futuro del Trabajo que Queremos (pp 95-113). Albacete: Bomarzo

UK Commission for Emploment and Skills (2014). The future of Work. Jobs and Skills in 2030. https://www.oitcinterfor.org/sites/default/files/file_publicacion/thefutureofwork. pdf [consulta 13 de diciembre de 2017].

Webster, J. (2016). "Microworkers of the Gig Economy: Separate and Precarious". New Labor Forum. 25 (3): 56-64.

Webster, J. y Randle, K. (2016). Virtual Workers and the Global Labour Market. London: Palgrave Macmillan.

World Economic Forum (2016). The Future of Jobs. Employment, Skills and Workforce Strategy for the Fourth Industrial Revolution. http://www3.weforum.org/docs/WEF_Future_of_Jobs.pdf [consulta 13 de diciembre de 2017]. 